\title{
Upper class of type I on coupled coincidence point results for some contractions in partially ordered JS-metric spaces
}

\author{
A.H. Ansari' ${ }^{1}$ A. Khemphet ${ }^{2,3}$, N. Phudolsitthiphat ${ }^{2,3^{*}}$ (D) and A. Wiriyapongsanon ${ }^{4}$
}

\section{"Correspondence:}

narawadee_n@hotmail.co.th ${ }^{2}$ Research Center in Mathematics and Applied Mathematics, Department of Mathematics, Faculty of Science, Chiang Mai University, Chiang Mai, Thailand Full list of author information is available at the end of the article

\begin{abstract}
In this research work, the necessary and sufficient conditions of a coupled coincidence point of certain type of generalized contractions are explored. These results are considered under JS-metric spaces equipped with a partial order. Moreover, examples satisfying theorems are given. Finally, an application to a system of integral equations is obtained using our results. In addition, an example of the system is provided.
\end{abstract}

MSC: Primary $47 \mathrm{H} 10$; secondary $54 \mathrm{H} 25$

Keywords: Coupled coincidence point; Upper class; JS-metric space; Partially ordered set; Geraghty

\section{Introduction}

For a pair of mappings $S: X \times X \rightarrow X$ and $t: X \rightarrow X$ defined on a nonempty set $X$, we would like to find a coupled coincidence point of $S$ and $t$, that is, an element $(x, y) \in X \times X$ satisfying

$$
t x=S(x, y) \text { and } \quad t y=S(y, x) .
$$

In 2015, Kadelburg et al. [4] obtained a result for some contraction mappings on a metric that defined a complete space that is partially ordered. In the same year, Jleli and Samet [3] extended the concept of a metric space to another space called a JS-metric space. The class of such metric spaces covers the class of standard metric, $b$-metric, and dislocated metric spaces. The author also proved the Banach contraction principle and Ćirićs fixed point theorem in JS-metric spaces. Now, let us recall the definition. Assume a mapping $\mathcal{D}: X \times X \rightarrow[0,+\infty]$ satisfying for every $x, y \in X:$

$\left(\mathcal{D}_{1}\right)$ if $\mathcal{D}(x, y)=0$ then $x=y$,

$\left(\mathcal{D}_{2}\right) \mathcal{D}(x, y)=\mathcal{D}(y, x)$

$\left(\mathcal{D}_{3}\right)$ there exists $K>0$ so that

$$
\lim _{n \rightarrow \infty} \mathcal{D}\left(x_{n}, x\right)=0 \quad \text { implies } \mathcal{D}(x, y) \leq K \limsup _{n \rightarrow \infty} \mathcal{D}\left(x_{n}, y\right) .
$$

(c) The Author(s) 2019. This article is licensed under a Creative Commons Attribution 4.0 International License, which permits use sharing, adaptation, distribution and reproduction in any medium or format, as long as you give appropriate credit to the original author(s) and the source, provide a link to the Creative Commons licence, and indicate if changes were made.The images or other third party material in this article are included in the article's Creative Commons licence, unless indicated otherwise in a credit line to the material. If material is not included in the article's Creative Commons licence and your intended use is not permitted by statutory regulation or exceeds the permitted use, you will need to obtain permission directly from the copyright holder.To view a copy of this licence, visit http://creativecommons.org/licenses/by/4.0/. 
Then we call $\mathcal{D}$ a JS-metric on $X$ and $(X, \mathcal{D})$ a JS-metric space.

As in classical metric spaces, similar definitions for sequences in JS-metric spaces are defined in the usual way [3].

In 2017, Phudolsitthiphat and Wiriyapongsanon [6] extended the result in [4] to find a coupled coincidence point of $\alpha$-Geraghty contractions under JS-metric spaces. Next, Ansari and Shukla $[1,2]$ gave the idea of a relation between $\mathcal{F}$ and $h$, precisely, the pair $(\mathcal{F}, h)$ to be an upper class.

In this article, motivated by the discussion above, we obtain the existence results of a coupled coincidence point of Geraghty-type contraction mappings with the pair $(\mathcal{F}, h)$ being an upper class of type I in partially ordered JS-metric spaces. Furthermore, examples of our theorems are given. Additionally, our results can be applied to a system of integral equations. The existence theorems of a solution to the system are given together with an example supporting the theorem.

\section{Main results}

Let us begin by recalling some notations and definitions needed in this section. Assume that $X$ is a nonempty set. Suppose that $S: X \times X \rightarrow X$ and $t: X \rightarrow X$ are two mappings. Then $t$ commutes with $S$ if

$$
t S(x, y)=S(t x, t y) \quad \text { for every } x, y \in X .
$$

For a partial order $\preceq$, define $E_{\preceq}=\{(x, y) \in X \times X: x \preceq y\}$ (see [3]). Then $S$ has the $\preceq-t$ monotone property if, for every $x, y \in X$,

$$
x_{1}, x_{2} \in X,\left(t x_{1}, t x_{2}\right) \in E_{\leq} \quad \Longrightarrow \quad\left(S\left(x_{1}, y\right), S\left(x_{2}, y\right)\right) \in E_{\leq}
$$

and

$$
y_{1}, y_{2} \in X,\left(t y_{1}, t y_{2}\right) \in E_{\leq} \quad \Longrightarrow \quad\left(S\left(x, y_{1}\right), S\left(x, y_{2}\right)\right) \in E_{ц} .
$$

Next, we give the definition of triangular $\alpha$-admissible property, which is needed later in this section.

Definition 2.1 Suppose that $S: X \times X \rightarrow X, t: X \rightarrow X$ and $\alpha: X^{2} \times X^{2} \rightarrow[0,+\infty]$ satisfy the following:

(i) if $\alpha((t x, t y),(t u, t v)) \geq 1$, then $\alpha((S(x, y), S(y, x)),(S(u, v), S(v, u))) \geq 1$,

(ii) if $\alpha((t x, t y),(t u, t v)) \geq 1$ and $\alpha((t u, t v),(S(u, v), S(v, u))) \geq 1$, then

$$
\alpha((t x, t y),(S(u, v), S(v, u))) \geq 1 .
$$

Then $S$ and $t$ are said to be triangular $\alpha$-admissible.

Now, let $\Theta^{\prime}$ consist of all $\theta:[0,+\infty] \times[0,+\infty] \rightarrow[0,1)$ so that all the following hold:

$\left(\theta_{1}\right) \theta(s, t)=\theta(t, s)$ for any $s, t \in[0,+\infty]$,

$\left(\theta_{2}\right)$ for $\left\{s_{n}\right\},\left\{t_{n}\right\} \subseteq[0,+\infty]$,

$$
\lim _{n \rightarrow \infty} \theta\left(s_{n}, t_{n}\right)=1 \text { implies } \lim _{n \rightarrow \infty} s_{n}=\lim _{n \rightarrow \infty} t_{n}=0 .
$$


Moreover, refer to [1, 2], the definition of an upper class of type I is extended as follows.

Definition 2.2 Given two mappings $h:[0,+\infty] \times[0,+\infty] \rightarrow \mathbb{R} \cup\{+\infty\}$ and $\mathcal{F}:[0,+\infty] \times$ $[0,+\infty] \rightarrow \mathbb{R} \cup\{+\infty\}$, define an upper class of type I to be the pair $(\mathcal{F}, h)$ satisfying that, for all $s, t, x, y \in[0,+\infty]$,

(i) $h(1, y) \leq h(x, y)$ whenever $1 \leq x$,

(ii) $\mathcal{F}(s, t) \leq \mathcal{F}(1, t)$ whenever $0 \leq s \leq 1$,

(iii) $y \leq s t$ whenever $h(1, y) \leq \mathcal{F}(s, t)$.

Example 2.3 For $l>1, m, n \in \mathbb{N}$, each pair $(\mathcal{F}, h)$ is an upper class of type I, where $h:[0,+\infty] \times[0,+\infty] \rightarrow \mathbb{R} \cup\{+\infty\}$ and $\mathcal{F}:[0,+\infty] \times[0,+\infty] \rightarrow \mathbb{R} \cup\{+\infty\}$ are defined as follows:

(a) $h(x, y)=\left\{\begin{array}{ll}(y+l)^{x} & \text { if } x, y \in[0,+\infty), \\ +\infty & \text { otherwise, }\end{array}\right.$ and $\mathcal{F}(s, t)= \begin{cases}s t+l & \text { if } x \in[0,+\infty) \\ +\infty & \text { otherwise }\end{cases}$

(b) $h(x, y)=\left\{\begin{array}{ll}(x+l)^{y} & \text { if } x, y \in[0,+\infty),\end{array}\right.$ and $\mathcal{F}(s, t)= \begin{cases}(1+l)^{s t} & \text { if } x \in[0,+\infty) \text {, }\end{cases}$

(c) $h(x, y)=\left\{\begin{array}{ll}y & \text { if } x, y \in[0,+\infty), \\ +\infty & \text { otherwise, }\end{array}\right.$ and $\mathcal{F}(s, t)= \begin{cases}s t & \text { if } x \in[0,+\infty) \\ +\infty & \text { otherwise }\end{cases}$

(d) $h(x, y)=\left\{\begin{array}{ll}x^{m} y^{n} & \text { if } x, y \in[0,+\infty), \\ +\infty & \text { otherwise, }\end{array}\right.$ and $\mathcal{F}(s, t)= \begin{cases}s^{n} t^{n} & \text { if } x \in[0,+\infty) \\ +\infty & \text { otherwise }\end{cases}$

To introduce our first theorem, for convenience purposes, assume all of the following throughout this section. Assume that $(X, \mathcal{D}, \preceq)$ is a complete partially ordered JS-metric space. Define $S: X \times X \rightarrow X$ and $t: X \rightarrow X$ to have the properties as follows:

(i) $S\left(X^{2}\right) \subseteq t(X)$,

(ii) $S$ is $\preceq$ - $t$-monotone,

(iii) $t$ is $\mathcal{D}$-continuous and commutes with $S$.

Now, we state the first main theorem. This result is a generalized version for coupled coincidence points of [6, Theorem 3.1].

\section{Theorem 2.4 Suppose that each statement is true:}

(i) For any upper class $(\mathcal{F}, h)$ of type $I$, there are $\alpha: X^{2} \times X^{2} \rightarrow[0,+\infty]$ and $\theta \in \Theta^{\prime}$ satisfying, for any $(t x, t u) \in E_{\leq},(t y, t v) \in E_{\leq}$, the inequality is obtained:

$$
\begin{aligned}
& h(\alpha((t x, t y),(t u, t v)), \mathcal{D}(S(x, y), S(u, v))) \\
& \quad \leq \mathcal{F}(\theta(\mathcal{D}(t x, t u), \mathcal{D}(t y, t v)), M((t x, t u),(t y, t v)))
\end{aligned}
$$

when

$$
\begin{aligned}
M((t x, t u),(t y, t v))= & \max \{\mathcal{D}(t x, t u), \mathcal{D}(t y, t v), \mathcal{D}(t x, S(x, y)), \\
& \mathcal{D}(t y, S(y, x)), \mathcal{D}(t u, S(u, v)), \mathcal{D}(t v, S(v, u))\}
\end{aligned}
$$

(ii) $S$ and $t$ are triangular $\alpha$-admissible, and there are $x_{0}, y_{0} \in X$, $\left(t x_{0}, S\left(x_{0}, y_{0}\right)\right),\left(t y_{0}, S\left(y_{0}, x_{0}\right)\right) \in E_{\leq}$so that

$$
\begin{aligned}
& \alpha\left(\left(t x_{0}, t y_{0}\right),\left(S\left(x_{0}, y_{0}\right), S\left(y_{0}, x_{0}\right)\right)\right) \geq 1 \quad \text { and } \\
& \alpha\left(\left(t y_{0}, t x_{0}\right),\left(S\left(y_{0}, x_{0}\right), S\left(x_{0}, y_{0}\right)\right)\right) \geq 1
\end{aligned}
$$


(iii) If $\lim _{n \rightarrow \infty} \mathcal{D}\left(t x_{n}, t x_{n+1}\right)=0$ and $\lim _{n \rightarrow \infty} \mathcal{D}\left(t y_{n}, t y_{n+1}\right)=0$, then

$$
\sup \left\{\mathcal{D}\left(t x_{0}, t x_{n}\right), \mathcal{D}\left(t y_{0}, t y_{n}\right): n \in \mathbb{N}\right\}<\infty
$$

where $\left\{x_{n}\right\}$ and $\left\{y_{n}\right\}$ are sequences in $X$;

(iv) $S$ is $\mathcal{D}$-continuous.

Then a coupled coincidence point of t and $S$ exists.

Proof Suppose that $x_{0}$ and $y_{0}$ are elements in $X$ satisfying assumption (ii). Since $S\left(X^{2}\right) \subseteq$ $t(X)$, we can choose $x_{1}, y_{1} \in X$ such that $t x_{1}=S\left(x_{0}, y_{0}\right)$ and $t y_{1}=S\left(y_{0}, x_{0}\right)$. Similarly, $t x_{2}=$ $S\left(x_{1}, y_{1}\right)$ and $t y_{2}=S\left(y_{1}, x_{1}\right)$ for some $x_{2}, y_{2} \in X$. Continue this argument. Then $\left\{x_{n}\right\}$ and $\left\{y_{n}\right\}$ are obtained with

$$
t x_{n+1}=S\left(x_{n}, y_{n}\right) \text { and } t y_{n+1}=S\left(y_{n}, x_{n}\right)
$$

Suppose that $t x_{n_{0}+1}=t x_{n_{0}}$ and $t y_{n_{0}+1}=t y_{n_{0}}$ for some natural number $n_{0}$. Then a coupled coincidence point of $t$ and $S$ exists, i.e., $\left(x_{n_{0}}, y_{n_{0}}\right)$, and so we are done. Assume that

$$
t x_{n+1} \neq t x_{n} \quad \text { or } \quad t y_{n+1} \neq t y_{n}
$$

for each positive integer $n$. By condition (ii),

$$
\left(t x_{0}, t x_{1}\right) \in E_{\preceq} \quad \text { and } \quad\left(t y_{0}, t y_{1}\right) \in E_{\preceq} .
$$

Since $S$ is $\preceq-t$-monotone,

$$
\left(S\left(x_{0}, y_{0}\right), S\left(x_{1}, y_{1}\right)\right) \in E_{\preceq} \quad \text { and } \quad\left(S\left(y_{0}, x_{0}\right), S\left(y_{1}, x_{1}\right)\right) \in E_{\preceq} .
$$

That is,

$$
\left(t x_{1}, t x_{2}\right) \in E_{\preceq} \quad \text { and } \quad\left(t y_{1}, t y_{2}\right) \in E_{\preceq} .
$$

Repeat this process, we have that

$$
\left(t x_{n}, t x_{n+1}\right) \in E_{\preceq} \quad \text { and } \quad\left(t y_{n}, t y_{n+1}\right) \in E_{\preceq} \quad \text { for all } n \in \mathbb{N} \text {. }
$$

By transitivity of $\preceq$, we get that

$$
\left(t x_{n}, t x_{n+m}\right) \in E_{\preceq} \quad \text { and } \quad\left(t y_{n}, t y_{n+m}\right) \in E_{\preceq} \quad \text { for all } n, m \in \mathbb{N} \text {. }
$$

From assumption (ii),

$$
\alpha\left(\left(t x_{0}, t y_{0}\right),\left(t x_{1}, t y_{1}\right)\right)=\alpha\left(\left(t x_{0}, t y_{0}\right),\left(S\left(x_{0}, y_{0}\right), S\left(y_{0}, x_{0}\right)\right)\right) \geq 1
$$

Since $S$ and $t$ are $\alpha$-admissible,

$$
\alpha\left(\left(t x_{1}, t y_{1}\right),\left(t x_{2}, t y_{2}\right)\right)=\alpha\left(\left(S\left(x_{0}, y_{0}\right), S\left(y_{0}, x_{0}\right)\right),\left(S\left(x_{1}, y_{1}\right), S\left(y_{1}, x_{1}\right)\right)\right) \geq 1
$$


Thus, by mathematical induction, we obtain that

$$
\alpha\left(\left(t x_{n}, t y_{n}\right),\left(t x_{n+1}, t y_{n+1}\right)\right) \geq 1 \quad \text { for all } n \in \mathbb{N} \text {. }
$$

With the same process as above, it can be concluded that

$$
\alpha\left(\left(t y_{n}, t x_{n}\right),\left(t y_{n+1}, t x_{n+1}\right)\right) \geq 1 \quad \text { for all } n \in \mathbb{N} \text {. }
$$

Since $S$ and $t$ are triangular $\alpha$-admissible,

$$
\begin{aligned}
& \alpha\left(\left(t x_{n}, t y_{n}\right),\left(t x_{n+m}, t y_{n+m}\right)\right) \geq 1 \quad \text { and } \\
& \alpha\left(\left(t y_{n}, t x_{n}\right),\left(t y_{n+m}, t x_{n+m}\right)\right) \geq 1 \quad \text { for all } n, m \in \mathbb{N} .
\end{aligned}
$$

Next, we prove the following claims.

Claim $1 . \lim _{n \rightarrow \infty} \mathcal{D}\left(t x_{n}, t x_{n+1}\right)=0$ and $\lim _{n \rightarrow \infty} \mathcal{D}\left(t y_{n}, t y_{n+1}\right)=0$.

To prove by contradiction, assume that either $\lim _{n \rightarrow \infty} \mathcal{D}\left(t x_{n}, t x_{n+1}\right) \neq 0$ or

$\lim _{n \rightarrow \infty} \mathcal{D}\left(t y_{n}, t y_{n+1}\right) \neq 0$. Then there exists $\varepsilon>0$ for which we can obtain a subsequence $\left\{n_{k}\right\}$ such that $n_{k} \geq k$ and

$$
\epsilon \leq \max \left\{\mathcal{D}\left(t x_{n_{k}}, t x_{n_{k}+1}\right), \mathcal{D}\left(t y_{n_{k}}, t y_{n_{k}+1}\right)\right\} .
$$

Consider

$$
\begin{aligned}
h( & \left.1, \mathcal{D}\left(t x_{n_{k}}, t x_{n_{k}+1}\right)\right) \\
& =h\left(1, \mathcal{D}\left(S\left(x_{n_{k}-1}, y_{n_{k}-1}\right), S\left(x_{n_{k}}, y_{n_{k}}\right)\right)\right) \\
& \leq h\left(\alpha\left(\left(t x_{n_{k}-1}, t y_{n_{k}-1}\right),\left(t x_{n_{k}}, t y_{n_{k}}\right)\right), \mathcal{D}\left(S\left(x_{n_{k}-1}, y_{n_{k}-1}\right), S\left(x_{n_{k}}, y_{n_{k}}\right)\right)\right) \\
& \leq \mathcal{F}\left(\theta\left(\mathcal{D}\left(t x_{n_{k}-1}, t x_{n_{k}}\right), \mathcal{D}\left(t y_{n_{k}-1}, t y_{n_{k}}\right)\right), M\left(\left(t x_{n_{k}-1}, t x_{n_{k}}\right),\left(t y_{n_{k}-1}, t y_{n_{k}}\right)\right)\right)
\end{aligned}
$$

and

$$
\begin{aligned}
h( & \left., \mathcal{D}\left(t y_{n_{k}}, t y_{n_{k}+1}\right)\right) \\
& =h\left(1, \mathcal{D}\left(S\left(y_{n_{k}-1}, x_{n_{k}-1}\right), S\left(y_{n_{k}}, x_{n_{k}}\right)\right)\right) \\
& \leq h\left(1, \alpha\left(\left(t y_{n_{k}-1}, t x_{n_{k}-1}\right),\left(t y_{n_{k}}, t x_{n_{k}}\right)\right) \mathcal{D}\left(S\left(y_{n_{k}-1}, x_{n_{k}-1}\right), S\left(y_{n_{k}}, x_{n_{k}}\right)\right)\right) \\
& \leq \mathcal{F}\left(\theta\left(\mathcal{D}\left(t y_{n_{k}-1}, t y_{n_{k}}\right), \mathcal{D}\left(t x_{n_{k}-1}, t x_{n_{k}}\right)\right), M\left(\left(t y_{n_{k}-1}, t y_{n_{k}}\right),\left(t x_{n_{k}-1}, t x_{n_{k}}\right)\right)\right) .
\end{aligned}
$$

These two inequalities imply that

$$
\begin{aligned}
& \mathcal{D}\left(t x_{n_{k}}, t x_{n_{k}+1}\right) \\
& \quad \leq \theta\left(\mathcal{D}\left(t x_{n_{k}-1}, t x_{n_{k}}\right), \mathcal{D}\left(t y_{n_{k}-1}, t y_{n_{k}}\right)\right) M\left(\left(t x_{n_{k}-1}, t x_{n_{k}}\right),\left(t y_{n_{k}-1}, t y_{n_{k}}\right)\right)
\end{aligned}
$$

and

$$
\mathcal{D}\left(t y_{n_{k}}, t y_{n_{k}+1}\right)
$$




$$
\leq \theta\left(\mathcal{D}\left(t y_{n_{k}-1}, t y_{n_{k}}\right), \mathcal{D}\left(t x_{n_{k}-1}, t x_{n_{k}}\right)\right) M\left(\left(t y_{n_{k}-1}, t y_{n_{k}}\right),\left(t x_{n_{k}-1}, t x_{n_{k}}\right)\right) .
$$

Since $\theta(s, t) \in[0,1)$ for any $s, t \in[0,+\infty]$,

$$
\begin{aligned}
M\left(\left(t x_{n_{k}-1}, t x_{n_{k}}\right),\left(t y_{n_{k}-1}, t y_{n_{k}}\right)\right) & =M\left(\left(t y_{n_{k}-1}, t y_{n_{k}}\right),\left(t x_{n_{k}-1}, t x_{n_{k}}\right)\right) \\
& =\max \left\{\mathcal{D}\left(t x_{n_{k}-1}, t x_{n_{k}}\right), \mathcal{D}\left(t y_{n_{k}-1}, t y_{n_{k}}\right)\right\} .
\end{aligned}
$$

From (2.1), (2.2), and (2.3),

$$
\begin{aligned}
& \max \left\{\mathcal{D}\left(t x_{n_{k}}, t x_{n_{k}+1}\right), \mathcal{D}\left(t y_{n_{k}}, t y_{n_{k}+1}\right)\right\} \\
& \quad \leq \theta\left(\mathcal{D}\left(t x_{n_{k}-1}, t x_{n_{k}}\right), \mathcal{D}\left(t y_{n_{k}-1}, t y_{n_{k}}\right)\right) \max \left\{\mathcal{D}\left(t x_{n_{k}-1}, t x_{n_{k}}\right), \mathcal{D}\left(t y_{n_{k}-1}, t y_{n_{k}}\right)\right\}
\end{aligned}
$$

With this idea, we finally have the following inequality:

$$
\begin{aligned}
& \max \left\{\mathcal{D}\left(t x_{n_{k}}, t x_{n_{k}+1}\right), \mathcal{D}\left(t y_{n_{k}}, t y_{n_{k}+1}\right)\right\} \\
& \leq \prod_{i=1}^{n_{k}} \theta\left(\mathcal{D}\left(t x_{n_{k}-i}, t x_{n_{k}+1-i}\right), \mathcal{D}\left(t y_{n_{k}-i}, t y_{n_{k}+1-i}\right)\right) \max \left\{\mathcal{D}\left(t x_{0}, t x_{1}\right), \mathcal{D}\left(t y_{0}, t y_{1}\right)\right\}
\end{aligned}
$$

Then choose $i_{k}$ such that

$$
\begin{aligned}
& \theta\left.\mathcal{D}\left(t x_{n_{k}-i_{k}}, t x_{n_{k}+1-i_{k}}\right), \mathcal{D}\left(t y_{n_{k}-i_{k}}, t y_{n_{k}+1-i_{k}}\right)\right) \\
& \quad=\max _{1 \leq i \leq n_{k}}\left\{\theta\left(\mathcal{D}\left(t x_{n_{k}-i}, t x_{n_{k}+1-i}\right), \mathcal{D}\left(t y_{n_{k}-i}, t y_{n_{k}+1-i}\right)\right)\right\} .
\end{aligned}
$$

Define $\eta:=\lim \sup _{k \rightarrow \infty}\left\{\theta\left(\mathcal{D}\left(t x_{n_{k}-i_{k}}, t x_{n_{k}+1-i_{k}}\right), \mathcal{D}\left(t y_{n_{k}-i_{k}}, t y_{n_{k}+1-i_{k}}\right)\right)\right\}$.

If $\eta<1$, then

$$
\lim _{k \rightarrow \infty} \max \left\{\mathcal{D}\left(t x_{n_{k}}, t x_{n_{k}+1}\right), \mathcal{D}\left(t y_{n_{k}}, t y_{n_{k}+1}\right)\right\}=0
$$

This contradicts the assumption.

Assume that $\eta=1$. For convenience, we suppose that

$$
\lim _{k \rightarrow \infty} \theta\left(\mathcal{D}\left(t x_{n_{k}-i_{k}}, t x_{n_{k}+1-i_{k}}\right), \mathcal{D}\left(t y_{n_{k}-i_{k}}, t y_{n_{k}+1-i_{k}}\right)\right)=1 .
$$

Since $\theta \in \Theta^{\prime}$,

$$
\lim _{k \rightarrow \infty} \mathcal{D}\left(t x_{n_{k}-i_{k}}, t x_{n_{k}+1-i_{k}}\right)=0 \quad \text { and } \quad \lim _{k \rightarrow \infty} \mathcal{D}\left(t y_{n_{k}-i_{k}}, t y_{n_{k}+1-i_{k}}\right)=0
$$

That is, there exists $k_{0} \in \mathbb{N}$ such that

$$
\mathcal{D}\left(t x_{n_{k_{0}}-i_{k_{0}}}, t x_{n_{k_{0}}+1-i_{k_{0}}}\right)<\frac{\epsilon}{2} \quad \text { and } \quad \mathcal{D}\left(t y_{n_{k_{0}}-i_{k_{0}}}, t y_{n_{k_{0}}+1}-i_{k_{0}}\right)<\frac{\epsilon}{2} \text {. }
$$

Thus, we have that

$$
\epsilon \leq \max \left\{\mathcal{D}\left(t x_{n_{k_{0}}}, t x_{n_{k_{0}}+1}\right), \mathcal{D}\left(t y_{n_{k_{0}}}, t y_{n_{k_{0}}+1}\right)\right\}
$$




$$
\begin{aligned}
\leq & \prod_{j=1}^{i_{k_{0}}} \theta\left(\mathcal{D}\left(t x_{n_{k_{0}}-j}, t x_{n_{k_{0}}+1-j}\right), \mathcal{D}\left(t y_{n_{k_{0}}-j}, t y_{n_{k_{0}}+1-j}\right)\right) \\
& \times \max \left\{\mathcal{D}\left(t x_{n_{k_{0}}-i_{k_{0}}}, t x_{n_{k_{0}}+1-i_{k_{0}}}\right), \mathcal{D}\left(t y_{n_{k_{0}}-i_{k_{0}}}, t y_{n_{k_{0}}+1-i_{k_{0}}}\right)\right\} \\
< & \frac{\epsilon}{2} .
\end{aligned}
$$

This is a contradiction. Therefore, we conclude that

$$
\lim _{n \rightarrow \infty} \mathcal{D}\left(t x_{n}, t x_{n+1}\right)=0 \quad \text { and } \quad \lim _{n \rightarrow \infty} \mathcal{D}\left(t y_{n}, t y_{n+1}\right)=0
$$

Claim 2. $\left\{t x_{n}\right\}$ and $\left\{t y_{n}\right\}$ are $\mathcal{D}$-Cauchy sequences.

We suppose by contradiction that neither $\left\{t x_{n}\right\}$ nor $\left\{t y_{n}\right\}$ is a $\mathcal{D}$-Cauchy sequence. Consequently, subsequences $\left\{n_{k}\right\}$ and $\left\{m_{k}\right\}$ can be obtained, where $n_{k}, m_{k} \geq k$ for each $k \in \mathbb{N}$, and $\epsilon^{\prime} \leq \max \left\{\mathcal{D}\left(t x_{n_{k}}, t x_{n_{k}+m_{k}}\right), \mathcal{D}\left(t y_{n_{k}}, t y_{n_{k}+m_{k}}\right)\right\}$ for some $\epsilon^{\prime}>0$. Consider

$$
\begin{aligned}
h & \left(1, \mathcal{D}\left(t x_{n_{k}}, t x_{n_{k}+m_{k}}\right)\right) \\
& =h\left(1, \mathcal{D}\left(S\left(x_{n_{k}-1}, y_{n_{k}-1}\right), S\left(x_{n_{k}+m_{k}-1}, y_{n_{k}+m_{k}-1}\right)\right)\right) \\
& \leq h\left(\alpha\left(\left(t x_{n_{k}-1}, t y_{n_{k}-1}\right),\left(t x_{n_{k}+m_{k}-1}, t y_{n_{k}+m_{k}-1}\right)\right), \mathcal{D}\left(S\left(x_{n_{k}-1}, y_{n_{k}-1}\right), S\left(x_{n_{k}-1}, y_{n_{k}-1}\right)\right)\right) \\
& \leq \mathcal{F}\left(\theta\left(\mathcal{D}\left(t x_{n_{k}-1}, t x_{n_{k}+m_{k}-1}\right), \mathcal{D}\left(t y_{n_{k}-1}, t y_{n_{k}+m_{k}-1}\right)\right), M\left(\left(t x_{n_{k}-1}, t x_{n_{k}+m_{k}-1}\right),\left(t y_{n_{k}-1}, t y_{n_{k}+m_{k}-1}\right)\right)\right)
\end{aligned}
$$

and

$$
\begin{aligned}
h\left(1, \mathcal{D}\left(t y_{n_{k}}, t y_{n_{k}+m_{k}}\right)\right) \\
\quad=h\left(1, \mathcal{D}\left(S\left(y_{n_{k}-1}, x_{n_{k}-1}\right), S\left(y_{n_{k}+m_{k}-1}, x_{n_{k}+m_{k}-1}\right)\right)\right) \\
\quad \leq h\left(\alpha\left(\left(t y_{n_{k}-1}, t x_{n_{k}-1}\right),\left(t y_{n_{k}+m_{k}-1}, t x_{n_{k}+m_{k}-1}\right)\right), \mathcal{D}\left(S\left(y_{n_{k}-1}, x_{n_{k}-1}\right), S\left(y_{n_{k}+m_{k}-1}, x_{n_{k}+m_{k}-1}\right)\right)\right) \\
\quad \leq \mathcal{F}\left(\theta\left(\mathcal{D}\left(t x_{n_{k}-1}, t x_{n_{k}+m_{k}-1}\right), \mathcal{D}\left(t y_{n_{k}-1}, t y_{n_{k}+m_{k}-1}\right)\right), M\left(\left(t x_{n_{k}-1}, t x_{n_{k}+m_{k}-1}\right),\left(t y_{n_{k}-1}, t y_{n_{k}+m_{k}-1}\right)\right) .\right.
\end{aligned}
$$

These imply that

$$
\begin{aligned}
& \mathcal{D}\left(t x_{n_{k}}, t x_{n_{k}+m_{k}}\right) \\
& \leq \theta\left(\mathcal{D}\left(t x_{n_{k}-1}, t x_{n_{k}+m_{k}-1}\right), \mathcal{D}\left(t y_{n_{k}-1}, t y_{n_{k}+m_{k}-1}\right)\right) \\
&\left.\quad \times M\left(\left(t x_{n_{k}-1}, t x_{n_{k}+m_{k}-1}\right),\left(t y_{n_{k}-1}, t y_{n_{k}+m_{k}-1}\right)\right)\right)
\end{aligned}
$$

and

$$
\begin{aligned}
& \mathcal{D}\left(t y_{n_{k}}, t y_{n_{k}+m_{k}}\right) \\
& \leq \theta\left(\mathcal{D}\left(t x_{n_{k}-1}, t x_{n_{k}+m_{k}-1}\right), \mathcal{D}\left(t y_{n_{k}-1}, t y_{n_{k}+m_{k}-1}\right)\right) \\
& \quad \times M\left(\left(t x_{n_{k}-1}, t x_{n_{k}+m_{k}-1}\right),\left(t y_{n_{k}-1}, t y_{n_{k}+m_{k}-1}\right)\right) .
\end{aligned}
$$

By (2.4), we have that

$$
M\left(\left(t x_{n_{k}-1}, t x_{n_{k}+m_{k}-1}\right),\left(t y_{n_{k}-1}, t y_{n_{k}+m_{k}-1}\right)\right)
$$




$$
\begin{aligned}
& =M\left(\left(t y_{n_{k}-1}, t y_{n_{k}+m_{k}-1}\right),\left(t x_{n_{k}-1}, t x_{n_{k}+m_{k}-1}\right)\right) \\
& =\max \left\{\mathcal{D}\left(t x_{n_{k}-1}, t x_{n_{k}+m_{k}-1}\right), \mathcal{D}\left(t y_{n_{k}-1}, t y_{n_{k}+m_{k}-1}\right)\right\} .
\end{aligned}
$$

From (2.5), (2.6), and (2.7), we obtain that

$$
\begin{aligned}
\max & \left\{\mathcal{D}\left(t x_{n_{k}}, t x_{n_{k}+m_{k}}\right), \mathcal{D}\left(t y_{n_{k}}, t y_{n_{k}+m_{k}}\right)\right\} \\
\leq & \theta\left(\mathcal{D}\left(t x_{n_{k}-1}, t x_{n_{k}+m_{k}-1}\right), \mathcal{D}\left(t y_{n_{k}-1}, t y_{n_{k}+m_{k}-1}\right)\right) \\
& \times \max \left\{\mathcal{D}\left(t x_{n_{k}-1}, t x_{n_{k}+m_{k}-1}\right), \mathcal{D}\left(t y_{n_{k}-1}, t y_{n_{k}+m_{k}-1}\right)\right\} .
\end{aligned}
$$

Therefore,

$$
\begin{aligned}
\max & \left\{\mathcal{D}\left(t x_{n_{k}}, t x_{n_{k}+m_{k}}\right), \mathcal{D}\left(t y_{n_{k}}, t y_{n_{k}+m_{k}}\right)\right\} \\
\leq & \prod_{i=1}^{n_{k}} \theta\left(\mathcal{D}\left(t x_{n_{k}-i}, t x_{n_{k}+m_{k}-i}\right), \mathcal{D}\left(t y_{n_{k}-i}, t y_{n_{k}+m_{k}-i}\right)\right) \\
& \times \max \left\{\mathcal{D}\left(t x_{0}, t x_{m_{k}}\right), \mathcal{D}\left(t y_{0}, t y_{m_{k}}\right)\right\}
\end{aligned}
$$

Choose $i_{k}$ such that

$$
\begin{aligned}
& \theta\left.\mathcal{D}\left(t x_{n_{k}-i_{k}}, t x_{n_{k}+m_{k}-i_{k}}\right), \mathcal{D}\left(t y_{n_{k}-i_{k}}, t y_{n_{k}+m_{k}-i_{k}}\right)\right) \\
& \quad=\max _{1 \leq i \leq n_{k}}\left\{\theta\left(\mathcal{D}\left(t x_{n_{k}-i}, t x_{n_{k}+m_{k}-i}\right), \mathcal{D}\left(t y_{n_{k}-i}, t y_{n_{k}+m_{k}-i}\right)\right)\right\} .
\end{aligned}
$$

Define $\eta:=\lim \sup _{k \rightarrow \infty}\left\{\theta\left(\mathcal{D}\left(t x_{n_{k}-i_{k}}, t x_{n_{k}+m_{k}-i_{k}}\right), \mathcal{D}\left(t y_{n_{k}-i_{k}}, t y_{n_{k}+m_{k}-i_{k}}\right)\right)\right\}$.

If $\eta<1$, then $\lim _{k \rightarrow \infty} \max \left\{\mathcal{D}\left(t x_{n_{k}}, t x_{n_{k}+m_{k}}\right), \mathcal{D}\left(t y_{n_{k}}, t y_{n_{k}+m_{k}}\right)\right\}=0$. This cannot be possible due to the assumption.

Assume that $\eta=1$. For convenience, suppose that

$$
\lim _{k \rightarrow \infty} \theta\left(\mathcal{D}\left(t x_{n_{k}-i_{k}}, t x_{n_{k}+m_{k}-i_{k}}\right), \mathcal{D}\left(t y_{n_{k}-i_{k}}, t y_{n_{k}+m_{k}-i_{k}}\right)\right)=1
$$

Since $\theta \in \Theta^{\prime}$ and (2.4),

$$
\lim _{k \rightarrow \infty} \mathcal{D}\left(t x_{n_{k}-i_{k}}, t x_{n_{k}+m_{k}-i_{k}}\right)=0 \quad \text { and } \quad \lim _{k \rightarrow \infty} \mathcal{D}\left(t y_{n_{k}-i_{k}}, t y_{n_{k}+m_{k}-i_{k}}\right)=0
$$

We obtain that

$$
\mathcal{D}\left(t x_{n_{k_{0}}-i_{k_{0}}}, t x_{n_{k_{0}}+m_{k_{0}}-i_{k_{0}}}\right)<\frac{\epsilon^{\prime}}{2} \quad \text { and } \quad \mathcal{D}\left(t y_{n_{k_{0}}-i_{k_{0}}}, t y_{n_{k_{0}}+m_{k_{0}}-i_{k_{0}}}\right)<\frac{\epsilon^{\prime}}{2}
$$

for some $k_{0} \in \mathbb{N}$. Thus, we have that

$$
\begin{aligned}
\epsilon^{\prime} \leq & \max \left\{\mathcal{D}\left(t x_{n_{k_{0}}}, t x_{n_{k_{0}}+m_{k_{0}}}\right), \mathcal{D}\left(t y_{n_{k_{0}}}, t y_{n_{k_{0}}+m_{k_{0}}}\right)\right\} \\
\leq & \prod_{j=1}^{i_{k_{0}}} \theta\left(\mathcal{D}\left(t x_{n_{k_{0}}-j}, t x_{n_{k_{0}}+m_{k_{0}}-j}\right), \mathcal{D}\left(t y_{n_{k_{0}}-j}, t y_{n_{k_{0}}+m_{k_{0}}-j}\right)\right) \\
& \quad \times \max \left\{\mathcal{D}\left(t x_{n_{k_{0}}-i_{k_{0}}}, t x_{n_{k_{0}}+m_{k_{0}}-i_{k_{0}}}\right), \mathcal{D} t\left(y_{n_{k_{0}}-i_{k_{0}}}, t y_{n_{k_{0}}+m_{k_{0}}-i_{k_{0}}}\right)\right\}
\end{aligned}
$$




$$
<\frac{\epsilon^{\prime}}{2}
$$

This is a contradiction. Thus, the sequences $\left\{t x_{n}\right\}$ and $\left\{t y_{n}\right\}$ must be $\mathcal{D}$-Cauchy. By completeness of $(X, \mathcal{D})$,

$$
\begin{aligned}
& \lim _{n \rightarrow \infty} \mathcal{D}\left(S\left(x_{n}, y_{n}\right), \omega\right)=\lim _{n \rightarrow \infty} \mathcal{D}\left(t x_{n}, \omega\right)=0, \\
& \lim _{n \rightarrow \infty} \mathcal{D}\left(S\left(y_{n}, x_{n}\right), \omega^{\prime}\right)=\lim _{n \rightarrow \infty} \mathcal{D}\left(t y_{n}, \omega^{\prime}\right)=0
\end{aligned}
$$

for some $\omega, \omega^{\prime} \in X$. By the continuity of $t$,

$$
\lim _{n \rightarrow \infty} \mathcal{D}\left(t\left(S\left(x_{n}, y_{n}\right)\right), t \omega\right)=0 \quad \text { and } \quad \lim _{n \rightarrow \infty} \mathcal{D}\left(t\left(S\left(y_{n}, x_{n}\right)\right), t \omega^{\prime}\right)=0
$$

By the continuity of $S$,

$$
\lim _{n \rightarrow \infty} \mathcal{D}\left(S\left(t x_{n}, t y_{n}\right), S\left(\omega, \omega^{\prime}\right)\right)=0 \quad \text { and } \quad \lim _{n \rightarrow \infty} \mathcal{D}\left(S\left(t y_{n}, t x_{n}\right), S\left(\omega^{\prime}, \omega\right)\right)=0
$$

Now, by the commutation between $t$ and $S$, it can be obtained that $t \omega=S\left(\omega, \omega^{\prime}\right)$ and $t \omega^{\prime}=$ $S\left(\omega^{\prime}, \omega\right)$. Therefore, a coupled coincidence point of $t$ and $S$ exists, namely $\left(\omega, \omega^{\prime}\right)$.

Corollary 2.5 Theorem 2.4 is valid when assumption $(i)$ is substituted by one of the following statements:

(i) There are $\theta \in \Theta^{\prime}$ and $l>1$ satisfying, for any $(t x, t u) \in E_{\leq},(t y, t v) \in E_{\leq}$, the inequality is obtained:

$$
\begin{aligned}
& (\mathcal{D}(S(x, y), S(u, v))+l)^{\alpha((t x, t y),(t u, t v))} \\
& \quad \leq \theta(\mathcal{D}(t x, t u), \mathcal{D}(t y, t v)) M((t x, t u),(t y, t v))+l
\end{aligned}
$$

when

$$
\begin{aligned}
M((t x, t u),(t y, t v))= & \max \{\mathcal{D}(t x, t u), \mathcal{D}(t y, t v), \mathcal{D}(t x, S(x, y)), \\
& \mathcal{D}(t y, S(y, x)), \mathcal{D}(t u, S(u, v)), \mathcal{D}(t v, S(v, u))\}
\end{aligned}
$$

(ii) There are $\theta \in \Theta^{\prime}$ and $l>1$ satisfying, for any $(t x, t u) \in E_{\preceq},(t y, t v) \in E_{\preceq}$, the inequality is obtained:

$$
(\alpha((t x, t y),(t u, t v))+l)^{\mathcal{D}(S(x, y), S(u, v))} \leq(1+l)^{\theta(\mathcal{D}(t x, t u), \mathcal{D}(t y, t v)) M((t x, t u),(t y, t v))}
$$

when

$$
\begin{aligned}
M((t x, t u),(t y, t v))= & \max \{\mathcal{D}(t x, t u), \mathcal{D}(t y, t v), \mathcal{D}(t x, S(x, y)), \\
& \mathcal{D}(t y, S(y, x)), \mathcal{D}(t u, S(u, v)), \mathcal{D}(t v, S(v, u))\}
\end{aligned}
$$

(iii) There is $\theta \in \Theta^{\prime}$ satisfying, for any $(t x, t u) \in E_{\leq},(t y, t v) \in E_{\leq}$, the inequality is obtained:

$$
\mathcal{D}(S(x, y), S(u, v)) \leq \theta(\mathcal{D}(t x, t u), \mathcal{D}(t y, t v)) M((t x, t u),(t y, t v))
$$


when

$$
\begin{aligned}
M((t x, t u),(t y, t v))= & \max \{\mathcal{D}(t x, t u), \mathcal{D}(t y, t v), \mathcal{D}(t x, S(x, y)), \\
& \mathcal{D}(t y, S(y, x)), \mathcal{D}(t u, S(u, v)), \mathcal{D}(t v, S(v, u))\}
\end{aligned}
$$

(iv) There is $\theta \in \Theta^{\prime}$ satisfying, for any $(t x, t u) \in E_{\preceq},(t y, t v) \in E_{\preceq}$, the inequality is obtained:

$$
\begin{aligned}
& (\alpha((t x, t y),(t u, t v)))^{m}(\mathcal{D}(S(x, y), S(u, v)))^{n} \\
& \quad \leq(\theta(\mathcal{D}(t x, t u) \mathcal{D}(t y, t v)))^{n}(M((t x, t u),(t y, t v)))^{n}
\end{aligned}
$$

for all positive integers $m, n$ when

$$
\begin{aligned}
M((t x, t u),(t y, t v))= & \max \{\mathcal{D}(t x, t u), \mathcal{D}(t y, t v), \mathcal{D}(t x, S(x, y)), \\
& \mathcal{D}(t y, S(y, x)), \mathcal{D}(t u, S(u, v)), \mathcal{D}(t v, S(v, u))\} .
\end{aligned}
$$

\section{Proof}

(i) Apply $h$ and $\mathcal{F}$ from Example 2.3(a) to Theorem 2.4.

(ii) Apply $h$ and $\mathcal{F}$ from Example 2.3(b) to Theorem 2.4.

(iii) Apply $h$ and $\mathcal{F}$ from Example 2.3(c) to Theorem 2.4.

(iv) Apply $h$ and $\mathcal{F}$ from Example 2.3(d) to Theorem 2.4.

\section{Remark 2.6}

(1) Corollary 2.5 holds for standard metric, $b$-metric, and dislocated metric spaces.

(2) Theorem 3.1 in [4] is a consequence of Corollary 2.5(iii).

(3) Theorem 3.1 in [6] is a special case of Corollary 2.5(iv) when $m=n=1$.

Example 2.7 Given $X=[0,+\infty]$ and $x, y \in X$, define $\mathcal{D}(x, y)=\max \{x, y\}$, and $S: X \times X \rightarrow X$ and $t: X \rightarrow X$ by

$$
S(x, y)=\left\{\begin{array}{ll}
\frac{x+y}{2} & \text { if } x, y \in[0,+\infty), \\
+\infty & \text { otherwise }
\end{array} \quad \text { and } \quad t x= \begin{cases}2 x & \text { if } x \in[0,+\infty) \\
+\infty & \text { otherwise }\end{cases}\right.
$$

Also, define $\alpha: X^{2} \times X^{2} \rightarrow[0,+\infty]$ by

$$
\alpha((x, y),(u, v))= \begin{cases}4 & \text { if } x \leq y \text { and } u \leq v \\ 0 & \text { otherwise }\end{cases}
$$

Let $x \leq u$ and $y \leq v$. Then

$$
\begin{aligned}
\mathcal{D}(S(x, y), S(u, v)) & =\max \left\{\frac{x+y}{2}, \frac{u+v}{2}\right\} \\
& \leq \frac{1}{2} \max \{2 u, 2 v\} \\
& =\theta(\mathcal{D}(t x, t u), \mathcal{D}(t y, t v)) M((t x, t u),(t y, t v))) .
\end{aligned}
$$


Thus, assumption (iii) of Corollary 2.5 holds for $\theta(k, l)=\frac{1}{2}$, where $k, l \in[0,+\infty]$. From Theorem 2.4, it can be easily shown that each property stated in the theorem holds. Hence, it follows that a coupled coincidence point of $t$ and $S$ exists.

In this example, observe that $\mathcal{D}$ is not a metric on $X$. Then Theorem 3.1 in [4] cannot be used to guarantee the existence of a coupled coincidence point for $t$ and $S$. Moreover, one of the assumptions of Theorems 3.1 and 3.2 in [6] fails as shown below. When $x \leq y \leq v$ and $x \leq u \leq v$, consider

$$
\begin{aligned}
\alpha((t x, t y),(t u, t v)) \mathcal{D}(S(x, y), S(u, v)) & =4 \max \left\{\frac{x+y}{2}, \frac{u+v}{2}\right\} \\
& =4\left(\frac{u+v}{2}\right) \\
& =2 u+2 v \\
& >\theta(\mathcal{D}(t x, t u), \mathcal{D}(t y, t v)) \cdot 2 v \\
& =\theta(\mathcal{D}(t x, t u), \mathcal{D}(t y, t v)) M((t x, t u),(t y, t v))
\end{aligned}
$$

for all $\theta \in \Theta^{\prime}$.

Define $\mathcal{F}^{\prime}$ to be the class of mappings $\beta:[0,+\infty] \rightarrow[0,1)$ such that

$$
\beta\left(t_{n}\right) \rightarrow 1 \quad \text { implies } t_{n} \rightarrow 0 \text { for all } t_{n} \in[0,+\infty]
$$

Next, we introduce our second main theorem which is a generalized version of [6, Theorem 3.2]. It can be remarked that the continuity of $S$ is not necessary to obtain the result.

Theorem 2.8 Suppose that each statement is true:

(i) For any upper class $(\mathcal{F}, h)$ of type $I$, there are $\alpha: X^{2} \times X^{2} \rightarrow[0,+\infty]$ and $\beta \in \mathcal{F}^{\prime}$ satisfying, for any $(t x, t u) \in E_{\preceq},(t y, t v) \in E_{\preceq}$, the inequality is obtained:

$$
\begin{aligned}
& h(\alpha((t x, t y),(t u, t v)), \mathcal{D}(S(x, y), S(u, v))) \\
& \quad \leq \mathcal{F}(\beta(M((t x, t u),(t y, t v))), M((t x, t u),(t y, t v)))
\end{aligned}
$$

when

$$
\begin{aligned}
M((t x, t u),(t y, t v))= & \max \{\mathcal{D}(t x, t u), \mathcal{D}(t y, t v), \mathcal{D}(t x, S(x, y)), \\
& \mathcal{D}(t y, S(y, x)), \mathcal{D}(t u, S(u, v)), \mathcal{D}(t v, S(v, u))\}
\end{aligned}
$$

(ii) $S$ and $t$ are triangular $\alpha$-admissible, and there are $x_{0}, y_{0} \in X$, $\left(t x_{0}, S\left(x_{0}, y_{0}\right)\right),\left(t y_{0}, S\left(y_{0}, x_{0}\right)\right) \in E_{\preceq}$ so that

$$
\begin{aligned}
& \alpha\left(\left(t x_{0}, t y_{0}\right),\left(S\left(x_{0}, y_{0}\right), S\left(y_{0}, x_{0}\right)\right)\right) \geq 1 \quad \text { and } \\
& \alpha\left(\left(t y_{0}, t x_{0}\right),\left(S\left(y_{0}, x_{0}\right), S\left(x_{0}, y_{0}\right)\right)\right) \geq 1
\end{aligned}
$$


(iii) If $\lim _{n \rightarrow \infty} \mathcal{D}\left(t x_{n}, t x_{n+1}\right)=0$ and $\lim _{n \rightarrow \infty} \mathcal{D}\left(t y_{n}, t y_{n+1}\right)=0$, then

$$
\sup \left\{\mathcal{D}\left(t x_{0}, t x_{n}\right), \mathcal{D}\left(t y_{0}, t y_{n}\right): n \in \mathbb{N}\right\}<\infty
$$

where $\left\{x_{n}\right\}$ and $\left\{y_{n}\right\}$ are sequences in $X$;

(iv) If $\left\{x_{n}\right\}$ and $\left\{y_{n}\right\}$ are sequences in $X$ with $\left(t x_{n}, t x_{n+1}\right),\left(t y_{n}, t y_{n+1}\right) \in E_{\preceq}$,

$$
\begin{aligned}
& \alpha\left(\left(t x_{n}, t y_{n}\right),\left(t x_{n+1}, t y_{n+1}\right)\right) \geq 1 \\
& \alpha\left(\left(t y_{n}, t x_{n}\right),\left(t y_{n+1}, t x_{n+1}\right)\right) \geq 1 \text { for all } n \in \mathbb{N},
\end{aligned}
$$

and $\lim _{n \rightarrow \infty} \mathcal{D}\left(t x_{n}, \omega\right)=0$ and $\lim _{n \rightarrow \infty} \mathcal{D}\left(t y_{n}, \omega^{\prime}\right)=0$, then $\left(t x_{n}, t \omega\right),\left(t y_{n}, t \omega^{\prime}\right) \in E_{\preceq}$,

$$
\begin{aligned}
& \alpha\left(\left(t x_{n}, t y_{n}\right),\left(t \omega, t \omega^{\prime}\right)\right) \geq 1 \\
& \alpha\left(\left(t y_{n}, t x_{n}\right),\left(t \omega^{\prime}, t \omega\right)\right) \geq 1 \quad \text { for all } n \in \mathbb{N} ;
\end{aligned}
$$

(v) There exists $0<C \leq 1$ such that

$$
\begin{aligned}
& \mathcal{D}\left(t \omega, S\left(\omega, \omega^{\prime}\right)\right) \leq C \underset{n \rightarrow \infty}{\limsup } \mathcal{D}\left(S\left(t x_{n}, t y_{n}\right), S\left(\omega, \omega^{\prime}\right)\right) \quad \text { and } \\
& \mathcal{D}\left(t \omega^{\prime}, S\left(\omega^{\prime}, \omega\right)\right) \leq C \limsup _{n \rightarrow \infty} \mathcal{D}\left(S\left(t y_{n}, t x_{n}\right), S\left(\omega^{\prime}, \omega\right)\right) .
\end{aligned}
$$

Then $t$ and $S$ have a coupled coincidence point.

Proof According to the statements in Theorem 2.4, the sequences $\left\{t x_{n}\right\}$ and $\left\{t y_{n}\right\}$ can be obtained. Moreover, by using $\beta(M((t x, t u),(t y, t v)))$ for $\theta(\mathcal{D}(t x, t u), \mathcal{D}(t y, t v))$ in Theorem 2.4, where $x, y, u, v \in X$, these two sequences are $\mathcal{D}$-Cauchy in a JS-metric space $(X, \mathcal{D})$ that is complete. As a result,

$$
\begin{aligned}
& \lim _{n \rightarrow \infty} \mathcal{D}\left(S\left(x_{n}, y_{n}\right), \omega\right)=\lim _{n \rightarrow \infty} \mathcal{D}\left(t x_{n}, \omega\right)=0, \\
& \lim _{n \rightarrow \infty} \mathcal{D}\left(S\left(y_{n}, x_{n}\right), \omega^{\prime}\right)=\lim _{n \rightarrow \infty} \mathcal{D}\left(t y_{n}, \omega^{\prime}\right)=0
\end{aligned}
$$

for some $\omega, \omega^{\prime} \in X$. By the continuity of $t$,

$$
\begin{aligned}
& \lim _{n \rightarrow \infty} \mathcal{D}\left(t S\left(x_{n}, y_{n}\right), t \omega\right)=\lim _{n \rightarrow \infty} \mathcal{D}\left(t t x_{n}, t \omega\right)=0, \\
& \lim _{n \rightarrow \infty} \mathcal{D}\left(t S\left(y_{n}, x_{n}\right), t \omega^{\prime}\right)=\lim _{n \rightarrow \infty} \mathcal{D}\left(t t y_{n}, t \omega^{\prime}\right)=0
\end{aligned}
$$

By assumptions (i), (ii), we have that

$$
\begin{aligned}
& h\left(1, \mathcal{D}\left(S\left(t x_{n}, t y_{n}\right), S\left(\omega, \omega^{\prime}\right)\right)\right) \\
& \quad \leq h\left(\alpha\left(\left(t t x_{n}, t t y_{n}\right),\left(t \omega, t \omega^{\prime}\right)\right), \mathcal{D}\left(S\left(t x_{n}, t y_{n}\right), S\left(\omega, \omega^{\prime}\right)\right)\right) \\
& \quad \leq \mathcal{F}\left(\beta\left(M\left(\left(t t x_{n}, t \omega\right),\left(t t y_{n}, t \omega^{\prime}\right)\right)\right), M\left(\left(t t x_{n}, t \omega\right),\left(t t y_{n}, t \omega^{\prime}\right)\right)\right)
\end{aligned}
$$


and

$$
\begin{aligned}
h\left(1, \mathcal{D}\left(S\left(t y_{n}, t x_{n}\right), S\left(\omega^{\prime}, \omega\right)\right)\right) \\
\quad \leq h\left(\alpha\left(\left(t t y_{n}, t t x_{n}\right),\left(t \omega^{\prime}, t \omega\right)\right), \mathcal{D}\left(S\left(t y_{n}, t x_{n}\right), S\left(\omega^{\prime}, \omega\right)\right)\right) \\
\quad \leq \mathcal{F}\left(\beta\left(M\left(\left(t t y_{n}, t \omega^{\prime}\right),\left(t t x_{n}, t \omega\right)\right)\right), M\left(\left(t t y_{n}, t \omega^{\prime}\right),\left(t t x_{n}, t \omega\right)\right)\right),
\end{aligned}
$$

where

$$
\begin{aligned}
M( & \left.\left(t t x_{n}, t \omega\right),\left(t t y_{n}, t \omega^{\prime}\right)\right) \\
= & M\left(\left(t t y_{n}, t \omega^{\prime}\right),\left(t t x_{n}, t \omega\right)\right) \\
= & \max \left\{\mathcal{D}\left(t t x_{n}, t \omega\right), \mathcal{D}\left(t t y_{n}, t \omega^{\prime}\right), \mathcal{D}\left(t t x_{n}, S\left(t x_{n}, t y_{n}\right)\right),\right. \\
& \left.\mathcal{D}\left(t t y_{n}, S\left(t y_{n}, t x_{n}\right)\right), \mathcal{D}\left(t \omega, S\left(\omega, \omega^{\prime}\right)\right), \mathcal{D}\left(t \omega^{\prime}, S\left(\omega^{\prime}, \omega\right)\right)\right\} .
\end{aligned}
$$

Consequently,

$$
\begin{aligned}
& \mathcal{D}\left(S\left(t x_{n}, t y_{n}\right), S\left(\omega, \omega^{\prime}\right)\right) \\
& \quad \leq \beta\left(M\left(\left(t t x_{n}, t \omega\right),\left(t t y_{n}, t \omega^{\prime}\right)\right)\right) M\left(\left(t t x_{n}, t \omega\right),\left(t t y_{n}, t \omega^{\prime}\right)\right)
\end{aligned}
$$

and

$$
\begin{aligned}
& \mathcal{D}\left(S\left(t y_{n}, t x_{n}\right), S\left(\omega^{\prime}, \omega\right)\right) \\
& \quad \leq \beta\left(M\left(\left(t t y_{n}, t \omega^{\prime}\right),\left(t t x_{n}, t \omega\right)\right)\right) M\left(\left(t t y_{n}, t \omega^{\prime}\right),\left(t t x_{n}, t \omega\right)\right) .
\end{aligned}
$$

Suppose that $t \omega \neq S\left(\omega, \omega^{\prime}\right)$ or $t \omega^{\prime} \neq S\left(\omega^{\prime}, \omega\right)$. That is,

$$
D:=\max \left\{\mathcal{D}\left(t \omega, S\left(\omega, \omega^{\prime}\right)\right), \mathcal{D}\left(t \omega^{\prime}, S\left(\omega^{\prime}, \omega\right)\right)\right\}>0 .
$$

By assumption (v), there exists $0<C \leq 1$ such that

$$
\mathcal{D}\left(t \omega, S\left(\omega, \omega^{\prime}\right)\right) \leq C \limsup _{n \rightarrow \infty} \mathcal{D}\left(S\left(t x_{n}, t y_{n}\right), S\left(\omega, \omega^{\prime}\right)\right) \leq C D
$$

and

$$
\mathcal{D}\left(t \omega^{\prime}, S\left(\omega^{\prime}, \omega\right)\right) \leq C \limsup _{n \rightarrow \infty} \mathcal{D}\left(S\left(t y_{n}, t x_{n}\right), S\left(\omega^{\prime}, \omega\right)\right) \leq C D
$$

Therefore,

$$
\begin{aligned}
D & =\max \left\{\mathcal{D}\left(t \omega, S\left(\omega, \omega^{\prime}\right)\right), \mathcal{D}\left(t \omega^{\prime}, S\left(\omega^{\prime}, \omega\right)\right)\right\} \\
& \leq C \limsup _{n \rightarrow \infty} \max \left\{\mathcal{D}\left(S\left(t x_{n}, t y_{n}\right), S\left(\omega, \omega^{\prime}\right)\right), \mathcal{D}\left(S\left(t y_{n}, t x_{n}\right), S\left(\omega^{\prime}, \omega\right)\right)\right\} \\
& \leq C D .
\end{aligned}
$$


Since $1 \leq \frac{1}{C}$, we get

$$
\begin{aligned}
D & \leq \frac{1}{C} D \\
& \leq \limsup _{n \rightarrow \infty} \max \left\{\mathcal{D}\left(S\left(t x_{n}, t y_{n}\right), S\left(\omega, \omega^{\prime}\right)\right), \mathcal{D}\left(S\left(t y_{n}, t x_{n}\right), S\left(\omega^{\prime}, \omega\right)\right)\right\} \\
& \leq D .
\end{aligned}
$$

It follows that $\limsup _{n \rightarrow \infty} \max \left\{\mathcal{D}\left(S\left(t x_{n}, t y_{n}\right), S\left(\omega, \omega^{\prime}\right)\right), \mathcal{D}\left(S\left(t y_{n}, t x_{n}\right), S\left(\omega^{\prime}, \omega\right)\right)\right\}=D$.

Then there exists a subsequence $\max \left\{\mathcal{D}\left(S\left(t x_{n_{k}}, t y_{n_{k}}\right), S\left(\omega, \omega^{\prime}\right)\right), \mathcal{D}\left(S\left(t y_{n_{k}}, t x_{n_{k}}\right), S\left(\omega^{\prime}, \omega\right)\right)\right\}$ such that

$$
\lim _{k \rightarrow \infty} \max \left\{\mathcal{D}\left(S\left(t x_{n_{k}}, t y_{n_{k}}\right), S\left(\omega, \omega^{\prime}\right)\right), \mathcal{D}\left(S\left(t y_{n_{k}}, t x_{n_{k}}\right), S\left(\omega^{\prime}, \omega\right)\right)\right\}=D
$$

Letting $k \rightarrow \infty$ in (2.8), we have that

$$
\lim _{n \rightarrow \infty} M\left(\left(t t x_{n_{k}}, t \omega\right),\left(t t y_{n_{k}}, t \omega^{\prime}\right)\right)=D
$$

From (2.9) and (2.10),

$$
\begin{aligned}
& \frac{\max \left\{\mathcal{D}\left(S\left(t x_{n_{k}}, t y_{n_{k}}\right), S\left(\omega, \omega^{\prime}\right)\right), \mathcal{D}\left(S\left(t y_{n_{k}}, t x_{n_{k}}\right), S\left(\omega^{\prime}, \omega\right)\right)\right\}}{M\left(\left(t t x_{n_{k}}, t \omega\right),\left(t t y_{n_{k}}, t \omega^{\prime}\right)\right)} \\
& \leq \beta\left(M\left(\left(t t x_{n_{k}}, t \omega\right),\left(t t y_{n_{k}}, t \omega^{\prime}\right)\right)\right) .
\end{aligned}
$$

Taking limit on both sides of the inequality, we obtain that

$$
\lim _{n \rightarrow \infty} \beta\left(M\left(\left(t t x_{n_{k}}, t \omega\right),\left(t t y_{n_{k}}, t \omega^{\prime}\right)\right)\right)=1
$$

Thus, $\lim _{n \rightarrow \infty} M\left(\left(t t x_{n_{k}}, t \omega\right),\left(t t y_{n_{k}}, t \omega^{\prime}\right)\right)=0$. This contradicts equation (2.11). Therefore, $t \omega=S\left(\omega, \omega^{\prime}\right)$ and $t \omega^{\prime}=S\left(\omega^{\prime}, \omega\right)$. Hence, $\left(\omega, \omega^{\prime}\right)$ is a coupled coincidence point of $t$ and $S$.

Corollary 2.9 Theorem 2.8 is valid when assumption $(i)$ is substituted by one of the following statements:

(i) There are $\beta \in \mathcal{F}^{\prime}$ and $l>1$ satisfying, for any $(t x, t u) \in E_{\leq},(t y, t v) \in E_{\leq}$, the inequality is obtained:

$$
\begin{aligned}
& (\mathcal{D}(S(x, y), S(u, v))+l)^{\alpha((t x, t y),(t u, t v))} \\
& \quad \leq \beta(M((t x, t u),(t y, t v))) M((t x, t u),(t y, t v))+l
\end{aligned}
$$

when

$$
\begin{aligned}
M((t x, t u),(t y, t v))= & \max \{\mathcal{D}(t x, t u), \mathcal{D}(t y, t v), \mathcal{D}(t x, S(x, y)), \\
& \mathcal{D}(t y, S(y, x)), \mathcal{D}(t u, S(u, v)), \mathcal{D}(t v, S(v, u))\}
\end{aligned}
$$


(ii) There are $\beta \in \mathcal{F}^{\prime}$ and $l>1$ satisfying, for any $(t x, t u) \in E_{\leq},(t y, t v) \in E_{\leq}$, the inequality is obtained:

$$
(\alpha((t x, t y),(t u, t v))+l)^{\mathcal{D}(S(x, y), S(u, v))} \leq(1+l)^{\beta(M((t x, t u),(t y, t v))) M((t x, t u),(t y, t v))}
$$

when

$$
\begin{aligned}
M((t x, t u),(t y, t v))= & \max \{\mathcal{D}(t x, t u), \mathcal{D}(t y, t v), \mathcal{D}(t x, S(x, y)), \\
& \mathcal{D}(t y, S(y, x)), \mathcal{D}(t u, S(u, v)), \mathcal{D}(t v, S(v, u))\}
\end{aligned}
$$

(iii) There is $\beta \in \mathcal{F}^{\prime}$ satisfying, for any $(t x, t u) \in E_{\leq},(t y, t v) \in E_{\leq}$, the inequality is obtained:

$$
\mathcal{D}(S(x, y), S(u, v)) \leq \beta(M((t x, t u),(t y, t v))) M((t x, t u),(t y, t v))
$$

when

$$
\begin{aligned}
M((t x, t u),(t y, t v))= & \max \{\mathcal{D}(t x, t u), \mathcal{D}(t y, t v), \mathcal{D}(t x, S(x, y)), \\
& \mathcal{D}(t y, S(y, x)), \mathcal{D}(t u, S(u, v)), \mathcal{D}(t v, S(v, u))\}
\end{aligned}
$$

(iv) There is $\beta \in \mathcal{F}^{\prime}$ satisfying, for any $(t x, t u) \in E_{\leq},(t y, t v) \in E_{\leq}$, the inequality is obtained:

$$
\begin{aligned}
& (\alpha((t x, t y),(t u, t v)))^{m}(\mathcal{D}(S(x, y), S(u, v)))^{n} \\
& \quad \leq(\beta(M((t x, t u),(t y, t v))))^{n}(M((t x, t u),(t y, t v)))^{n}
\end{aligned}
$$

for all positive integers $m, n$ when

$$
\begin{aligned}
M((t x, t u),(t y, t v))= & \max \{\mathcal{D}(t x, t u), \mathcal{D}(t y, t v), \mathcal{D}(t x, S(x, y)), \\
& \mathcal{D}(t y, S(y, x)), \mathcal{D}(t u, S(u, v)), \mathcal{D}(t v, S(v, u))\}
\end{aligned}
$$

\section{Proof}

(i) Apply $h$ and $\mathcal{F}$ from Example 2.3(a) to Theorem 2.8.

(ii) Apply $h$ and $\mathcal{F}$ from Example 2.3(b) to Theorem 2.8.

(iii) Apply $h$ and $\mathcal{F}$ from Example 2.3(c) to Theorem 2.8.

(iv) Apply $h$ and $\mathcal{F}$ from Example 2.3(d) to Theorem 2.8.

\section{Remark 2.10}

(1) Corollary 2.9 holds for standard metrics, $b$-metrics, and dislocated metrics.

(2) Theorem 3.2 in [6] is a consequence of Corollary 2.9(iv). Indeed, let $m=n=1$.

Example 2.11 Given $X=[0,+\infty]$ and $x, y \in X$, define $\mathcal{D}(x, y)=|x|+|y|$, and $S: X \times X \rightarrow X$ and $t: X \rightarrow X$ by

$$
S(x, y)=\left\{\begin{array}{ll}
\frac{|x-y|}{3} & \text { if } x, y \in[0,+\infty), \\
+\infty & \text { otherwise }
\end{array} \quad \text { and } \quad t x= \begin{cases}3 x & \text { if } x \in[0,+\infty) \\
+\infty & \text { otherwise }\end{cases}\right.
$$


Also, define $\alpha: X^{2} \times X^{2} \rightarrow[0,+\infty]$ by

$$
\alpha((x, y),(u, v))= \begin{cases}1 & \text { if } x \leq y \text { and } u \leq v \\ 0 & \text { otherwise. }\end{cases}
$$

Let $x \leq u$ and $y \leq v$. When $r>1$, consider

$$
\begin{aligned}
(\alpha((t x, t y),(t u, t v))+r)^{\mathcal{D}(S(x, y), S(u, v))} & \leq(1+r)^{\left(\frac{|x-y|}{3}+\frac{|u-v|}{3}\right)} \\
& \leq(1+r)^{\frac{1}{3}(|x|+|y|+|u|+|v|)} \\
& \leq(1+r)^{\frac{2}{3} \max \{|3 x|+|3 u|,|3 y|+|3 v|\}} \\
& =(1+r)^{\beta(M((t x, t u),(t y, t v))) M((t x, t u),(t y, t v))} .
\end{aligned}
$$

Thus, assumption (ii) of Corollary 2.9 holds for $\beta(t)=\frac{2}{3}$, where $t \in[0,+\infty]$. From Theorem 2.8 , it can be easily shown that each property stated in the theorem holds. Hence, it follows that a coupled coincidence point of $t$ and $S$ exists.

\section{Application}

In the real world, it has been known that many problems can be interpreted as a mathematical model. These models are written in several types of equations, for example, differential equations, integral equations, functional equations, partial differential equations. To find a solution to these problems, one has to solve the equations. There are some studies that propose a solution to such problems using fixed point theory, for example, see [5]. For this reason, it is our interest to apply our results to some problems. As a matter of fact, the theorems obtained in the previous section are used to state the necessary and sufficient conditions for the existence of a solution to a system of integral equations.

Given that $h:[0,1] \rightarrow \mathbb{R}, k_{i}:[0,1] \times[0,1] \rightarrow \mathbb{R}^{+}$and $f_{i}:[0,1] \times \mathbb{R} \rightarrow \mathbb{R}^{+}$are mappings, where $i=1,2$, consider the following system of integral equations:

$$
\begin{aligned}
& x(r)=h(r)+\int_{0}^{1} k_{1}(r, s) f_{1}(s, x(s)) d s \int_{0}^{1} k_{2}(r, s) f_{2}(s, y(s)) d s, \\
& y(r)=h(r)+\int_{0}^{1} k_{1}(r, s) f_{1}(s, y(s)) d s \int_{0}^{1} k_{2}(r, s) f_{2}(s, x(s)) d s,
\end{aligned}
$$

where $r \in[0,1]$. Assume that $X=C[0,1]$. Given that $\mathcal{D}(x, y)=\sup _{r \in[0,1]}|x(r)-y(r)|$ for $x, y \in X$, then $(X, \mathcal{D}, \preceq)$ is a complete partially ordered JS-metric space. Using results from the previous section, the existing theorems to a function satisfying system (3.1) can be obtained as follows.

Theorem 3.1 From the system of equations (3.1), define $S: X \times X \rightarrow X$ as

$$
S(x, y)(r)=h(r)+\int_{0}^{1} k_{1}(r, s) f_{1}(s, x(s)) d s \int_{0}^{1} k_{2}(r, s) f_{2}(s, y(s)) d s,
$$

where $r \in[0,1]$, and assume that

(i) $h:[0,1] \rightarrow \mathbb{R}, k_{i}:[0,1] \times[0,1] \rightarrow \mathbb{R}^{+}$and $f_{i}:[0,1] \times \mathbb{R} \rightarrow \mathbb{R}^{+}$are continuous, where $i=1,2$; 
(ii) $f_{1}\left(r, x_{1}(r)\right) \leq f_{1}\left(r, x_{2}(r)\right)$ and $f_{2}\left(r, x_{1}(r)\right) \leq f_{2}\left(r, x_{2}(r)\right)$ whenever $x_{1} \leq x_{2}$ for $x_{1}, x_{2} \in X$;

(iii) there are $K, L, M \in \mathbb{R}^{+}, 2 K L M<1, \int_{0}^{1} k_{i}(r, s) d s \leq K, \int_{0}^{1} k_{i}(r, s) f_{i}(s, x(s)) d s \leq M$, and

$$
\left|f_{i}(r, x(r))-f_{i}(r, u(r))\right| \leq L|x(r)-u(r)|
$$

where $0 \leq r \leq 1, x, u \in X$ and $i=1,2$;

(iv) there are $x_{0}, y_{0} \in X$ so that $x_{0} \leq S\left(x_{0}, y_{0}\right)$ and $y_{0} \leq S\left(y_{0}, x_{0}\right)$;

(v) if $x_{n}, y_{n} \in X$ such that $\lim _{n \rightarrow \infty}\left|x_{n}-x_{n+1}\right|=0$ and $\lim _{n \rightarrow \infty}\left|y_{n}-y_{n+1}\right|=0$, then $\sup \left\{\left|x_{0}-x_{n}\right|,\left|y_{0}-y_{n}\right|: n \in \mathbb{N}\right\}<\infty$, where $x_{0}$ and $y_{0}$ are defined as in (iv).

Then a solution to system (3.1) is obtained.

Proof Note that $S$ is $\mathcal{D}$-continuous. Define $t: X \rightarrow X$ by $t x=x$ for $x \in X$. Obviously, $S\left(X^{2}\right) \subseteq t(X)$ and $t$ is $\mathcal{D}$-continuous and commutes with $S$. Next, we show that $S$ is $\preceq$ $t$-monotone. Assume that $x_{1}, x_{2}, y \in X, x_{1} \preceq x_{2}$. According to assumption (ii),

$$
\begin{aligned}
S\left(x_{1}, y\right)(r) & =h(r)+\int_{0}^{1} k_{1}(r, s) f_{1}\left(s, x_{1}(s)\right) d s \int_{0}^{1} k_{2}(r, s) f_{2}(s, y(s)) d s \\
& \leq h(r)+\int_{0}^{1} k_{1}(r, s) f_{1}\left(s, x_{2}(s)\right) d s \int_{0}^{1} k_{2}(r, s) f_{2}(s, y(s)) d s \\
& =S\left(x_{2}, y\right)(r)
\end{aligned}
$$

and

$$
\begin{aligned}
S\left(y, x_{1}\right)(r) & =h(r)+\int_{0}^{1} k_{1}(r, s) f_{1}(s, y(s)) d s \int_{0}^{1} k_{2}(r, s) f_{2}\left(s, x_{1}(s)\right) d s \\
& \leq h(r)+\int_{0}^{1} k_{1}(r, s) f_{1}(s, y(s)) d s \int_{0}^{1} k_{2}(r, s) f_{2}\left(s, x_{2}(s)\right) d s \\
& =S\left(y, x_{2}\right)(r) .
\end{aligned}
$$

Thus, $S$ has the $\preceq-t$-monotone property.

Let $\alpha: X^{2} \times X^{2} \rightarrow[0,+\infty]$ be defined as $\alpha((x, y),(u, v))=1$ for any $(x, y),(u, v) \in X^{2}$. Clearly, $t$ and $S$ are triangular $\alpha$-admissible. In addition, it is easy to see that conditions (ii) and (iii) of Theorem 2.4 are satisfied from assumptions (iv) and (v), respectively.

At this point, to apply Theorem 2.4, only condition (i) is needed. However, this condition can be replaced by condition (iii) of Corollary 2.5. Now, we show that the latter condition is satisfied. Let $x, y, u, v \in X$. If $x \preceq u$ and $y \preceq v$, from assumption (iii),

$$
\begin{aligned}
& |S(x, y)(r)-S(u, v)(r)| \\
& =\mid \int_{0}^{1} k_{1}(r, s) f_{1}(s, x(s)) d s \int_{0}^{1} k_{2}(r, s) f_{2}(s, y(s)) d s \\
& \quad-\int_{0}^{1} k_{1}(r, s) f_{1}(s, u(s)) d s \int_{0}^{1} k_{2}(r, s) f_{2}(s, v(s)) d s \mid \\
& \leq\left|\int_{0}^{1} k_{1}(r, s) f_{1}(s, x(s)) d s \int_{0}^{1} k_{2}(r, s)\left[f_{2}(s, y(s))-f_{2}(s, v(s))\right] d s\right| \\
& \quad+\left|\int_{0}^{1} k_{1}(r, s)\left[f_{1}(s, x(s))-f_{1}(s, u(s))\right] d s \int_{0}^{1} k_{2}(r, s) f_{2}(s, v(s)) d s\right|
\end{aligned}
$$




$$
\begin{aligned}
& \leq K M L \sup _{r \in[0,1]}|y(r)-v(r)|+K M L \sup _{r \in[0,1]}|x(r)-u(r)| \\
& \leq 2 K M L \max \left\{\sup _{r \in[0,1]}|y(r)-v(r)|, \sup _{r \in[0,1]}|x(r)-u(r)|\right\} .
\end{aligned}
$$

Let $\theta=2 K M L<1$. Then $\theta \in \Theta^{\prime}$. Therefore, condition (iii) of Corollary 2.5 is obtained. Hence, a coupled fixed point of $S$ exists. Consequently, there is a solution to system (3.1).

An essential observation is that the given JS-metric $\mathcal{D}$ is, in fact, a metric. Now, define a new JS-metric $\mathcal{D}: X \times X \rightarrow[0, \infty]$ by $\mathcal{D}(x, y)=\sup _{r \in[0,1]}(|x(r)|+|y(r)|)$. It follows that this JS-metric $\mathcal{D}$ is not a metric. As a result, $(X, \mathcal{D}, \preceq)$ is complete partially ordered. Similarly, the existence of a solution to the system of integral equations can be guaranteed only if the integral equations are homogeneous.

Theorem 3.2 From the system of equations (3.1), let $S: X \times X \rightarrow X$ be defined as

$$
S(x, y)(r)=\int_{0}^{1} k_{1}(r, s) f_{1}(s, x(s)) d s \int_{0}^{1} k_{2}(r, s) f_{2}(s, y(s)) d s,
$$

where $r \in[0,1]$, and assume that

(i) $k_{i}:[0,1] \times[0,1] \rightarrow \mathbb{R}^{+}$and $f_{i}:[0,1] \times \mathbb{R} \rightarrow \mathbb{R}^{+}$are continuous, where $i=1,2$;

(ii) $f_{1}\left(r, x_{1}(r)\right) \leq f_{1}\left(r, x_{2}(r)\right)$ and $f_{2}\left(r, x_{1}(r)\right) \leq f_{2}\left(r, x_{2}(r)\right)$ whenever $x_{1} \leq x_{2}$ for $x_{1}, x_{2} \in X$;

(iii) there are $K, L, M \in \mathbb{R}^{+}, 2 K L M<1, \int_{0}^{1} k_{i}(r, s) d s \leq K, \int_{0}^{1} k_{i}(r, s) f_{i}(s, x(s)) d s \leq M$, and

$$
\left|f_{i}(r, x(r))\right|+\left|f_{i}(r, u(r))\right| \leq L(|x(r)|+|u(r)|)
$$

where $r \in[0,1], x, u \in X$ and $i=1,2$;

(iv) there are $x_{0}, y_{0} \in X$ so that $x_{0} \leq S\left(x_{0}, y_{0}\right)$ and $y_{0} \leq S\left(y_{0}, x_{0}\right)$.

Then a solution to system (3.1) is obtained when the integral equations are homogeneous.

Proof Similarly, let $t$ be an identity mapping on $X$. With this JS-metric $\mathcal{D}$, we get the $\mathcal{D}$ continuities of $t$ and $S$. Furthermore, it can be noticed that condition (iii) of Theorem 2.4 is always true for this JS-metric $\mathcal{D}$. Next, suppose $\alpha((x, y),(u, v))=1$ for any $(x, y),(u, v) \in$ $X^{2}$. Repeating the same arguments used in Theorem 3.1, it follows that all the conditions except condition (i) in Theorem 2.4 are valid.

To apply Theorem 2.4, our task is to prove condition (i) which, again, can be replaced by condition (iii) of Corollary 2.5. Let $x, y, u, v \in X$. If $x \preceq u$ and $y \preceq v$, by assumption (iii),

$$
\begin{aligned}
& |S(x, y)(r)|+|S(u, v)(r)| \\
& =\left|\int_{0}^{1} k_{1}(r, s) f_{1}(s, x(s)) d s \int_{0}^{1} k_{2}(r, s) f_{2}(s, y(s)) d s\right| \\
& \quad+\left|\int_{0}^{1} k_{1}(r, s) f_{1}(s, u(s)) d s \int_{0}^{1} k_{2}(r, s) f_{2}(s, v(s)) d s\right| \\
& \leq\left|\int_{0}^{1} k_{1}(r, s) f_{1}(s, x(s)) d s \int_{0}^{1} k_{2}(r, s)\left[f_{2}(s, y(s))+f_{2}(s, v(s))\right] d s\right|
\end{aligned}
$$




$$
\begin{aligned}
& +\left|\int_{0}^{1} k_{1}(r, s)\left[f_{1}(s, x(s))+f_{1}(s, u(s))\right] d s \int_{0}^{1} k_{2}(r, s) f_{2}(s, v(s)) d s\right| \\
\leq & K M L \sup _{r \in[0,1]}(|y(r)|+|v(r)|)+K M L \sup _{r \in[0,1]}(|x(r)|+|u(r)|) \\
\leq & 2 K M L \max \left\{\sup _{r \in[0,1]}(|y(r)|+|v(r)|), \sup _{r \in[0,1]}(|x(r)|+|u(r)|)\right\} .
\end{aligned}
$$

Likewise, let $\theta=2 K M L<1$, and so $\theta \in \Theta^{\prime}$. Then, we have condition (iii) of Corollary 2.5. As a result, there is a solution to system (3.1) when the integral equations are homogeneous.

Example 3.3 Consider the following system of integral equations:

$$
\begin{aligned}
& x(r)=\int_{0}^{1} s^{2} e^{-r^{2}} \cdot \frac{s^{3}}{1+s^{6}} \cdot \frac{|x(s)|}{1+|x(s)|} d s \int_{0}^{1} \frac{s^{2}}{1+r^{2}} \cdot \frac{1}{1+s^{3}} \cdot \frac{|y(s)|}{2+|y(s)|} d s, \\
& y(r)=\int_{0}^{1} s^{2} e^{-r^{2}} \cdot \frac{s^{3}}{1+s^{6}} \cdot \frac{|y(s)|}{1+|y(s)|} d s \int_{0}^{1} \frac{s^{2}}{1+r^{2}} \cdot \frac{1}{1+s^{3}} \cdot \frac{|x(s)|}{2+|x(s)|} d s,
\end{aligned}
$$

where $r \in[0,1]$. Define the following functions:

$$
\begin{aligned}
& k_{1}(r, s)=s^{2} e^{-r^{2}}, \\
& k_{2}(r, s)=\frac{s^{2}}{1+r^{2}}, \\
& f_{1}(s, x(s))=\frac{s^{3}}{1+s^{6}} \cdot \frac{|x(s)|}{1+|x(s)|}, \\
& f_{2}(s, x(s))=\frac{1}{1+s^{3}} \cdot \frac{|x(s)|}{2+|x(s)|},
\end{aligned}
$$

where $r, s \in[0,1]$. It can be seen that $k_{i}$ and $f_{i}$ are continuous, and $f_{i}(r, x) \geq 0$ for $i=1,2$. Moreover, $f_{i}(r, x(r)) \leq f_{i}(r, y(r))$ whenever $x \leq y$ for each $i=1,2$. In addition, note that condition (iv) of Theorem 3.2 holds for any nonpositive-valued mappings $x_{0}, y_{0}$. Finally, we have to show that this example satisfies condition (iii) of Theorem 3.2. Consider

$$
\begin{aligned}
& \left|f_{1}(r, x(r))\right|+\left|f_{1}(r, u(r))\right| \\
& \quad=\frac{r^{3}}{1+r^{6}} \cdot\left(\left|\frac{|x(r)|}{1+|x(r)|}\right|+\left|\frac{|u(r)|}{1+|u(r)|}\right|\right) \\
& \quad \leq \frac{1}{2}(|x(r)|+|u(r)|), \\
& \quad\left|f_{2}(r, y(r))\right|+\left|f_{2}(r, v(r))\right| \\
& \quad=\frac{1}{1+r^{3}} \cdot\left(\left|\frac{|y(r)|}{2+|y(r)|}\right|+\left|\frac{|v(r)|}{2+|v(r)|}\right|\right) \\
& \quad \leq \frac{1}{2}(|y(r)|+|v(r)|),
\end{aligned}
$$

then let $L=\frac{1}{2}$. Next,

$$
\int_{0}^{1} k_{1}(r, s) f_{1}\left(s, x_{1}(s)\right) d s \leq \int_{0}^{1} s^{2} e^{-r^{2}} \cdot \frac{s^{3}}{1+s^{6}} d s=\frac{1}{6} e^{-r^{2}} \ln 2 \leq \frac{1}{6} \ln 2,
$$




$$
\int_{0}^{1} k_{2}(r, s) f_{2}\left(s, x_{1}(s)\right) d s \leq \int_{0}^{1} \frac{s^{2}}{1+r^{2}} \cdot \frac{1}{1+s^{3}} d s=\frac{1}{1+r^{2}} \cdot \frac{1}{3} \ln 2 \leq \frac{1}{3} \ln 2 .
$$

Thus, setting $M=\frac{1}{3} \ln 2$, we get that $\int_{0}^{1} k_{i}(t, s) f_{i}\left(s, x_{1}(s)\right) d s \leq M$ for $i=1,2$. Since

$$
\begin{aligned}
& \int_{0}^{1} k_{1}(r, s) d s=\frac{1}{3} e^{-r^{2}} \leq \frac{1}{3}, \\
& \int_{0}^{1} k_{2}(r, s) d s=\frac{1}{3\left(1+r^{2}\right)} \leq \frac{1}{3},
\end{aligned}
$$

choose $K=\frac{1}{3}$. Consequently, $2 K L M<1$. Finally, each hypothesis in Theorem 3.2 is true. This leads to a conclusion that there is a solution to the system in $C[0,1]$.

\section{Acknowledgements}

The authors are grateful to the editors and referees for their valuable comments that helped to improve our work. Narawadee Phudolsitthiphat was supported by Chiang Mai University.

\section{Funding}

This research was supported by Chiang Mai University.

\section{Competing interests}

The authors have no conflict of interests regarding the publication of this paper.

\section{Authors' contributions}

The authors have contributed equally to this paper. All authors read and approved the final manuscript

\section{Author details}

${ }^{1}$ Department of Mathematics, Karaj Branch, Islamic Azad University, Karaj, Iran. ${ }^{2}$ Research Center in Mathematics and Applied Mathematics, Department of Mathematics, Faculty of Science, Chiang Mai University, Chiang Mai, Thailand. ${ }^{3}$ Data Science Research Center, Department of Mathematics, Faculty of Science, Chiang Mai University, Chiang Mai, Thailand.

${ }^{4}$ Faculty of Sport and Health Sciences, Thailand National Sports University Lampang Campus, Lampang, Thailand.

\section{Publisher's Note}

Springer Nature remains neutral with regard to jurisdictional claims in published maps and institutional affiliations.

Received: 15 February 2019 Accepted: 28 November 2019 Published online: 05 December 2019

\section{References}

1. Ansari, A.H.: Note on " $\alpha$-admissible mappings and related fixed point theorems". In: The 2nd Regional Conference on Mathematics and Applications, PNU, pp. 373-376 (2014)

2. Ansari, A.H., Shukla, S.: Some fixed point theorems for ordered $F-(\mathcal{F}, h)$-contraction and subcontractions in 0-f-orbitally complete partial metric spaces. J. Adv. Math. Stud. 9(1), 37-53 (2016)

3. Jeli, M., Samet, B.: A generalized metric space and related fixed point theorems. Fixed Point Theory Appl. 2015, 61 (2015)

4. Kadelburg, Z., Kumam, P., Radenović, S., Sintunavarat, W.: Common coupled fixed point theorems for Geraghty-type contraction mappings using monotone property. Fixed Point Theory Appl. 2015, 27 (2015)

5. Liang, M., Zhu, C., Wu, Z., Chen, C.: Some new coupled coincidence point and coupled fixed point results in partially ordered metric-like spaces and an application. J. Funct. Spaces 2018, Article ID 1378379 (2018)

6. Phudolsitthiphat, N., Wiriyapongsanon, A.: Coupled coincidence point results in partially ordered JS-metric spaces Novi Sad J. Math. 47(2), 173-185 (2017) 